\title{
18F-Choline PET/MR Can Detect and Delineate Local Recurrence After High-Intensity Focused Ultrasound Therapy of Prostate Cancer
}

Burger, Irene A ; Gafita, Andrei ; Müller, Julian ; Kranzbühler, Benedikt ; Donati, Olivio F ; Eberli, Daniel

\begin{abstract}
Restaging local recurrence after high-intensity focused ultra-sound (HIFU) is based on multiparametric MRI (mpMRI). However, postinterventional changes of the tissue, such as edema or hemorrhage, are limiting tumor detection on mpMRI. We present a case of a rising prostate-specific antigen values, negative mpMRI, and a Gleason score of $4+4$ on template biopsy after HIFU. On F-choline PET/MR, high focal uptake was detected at the location of positive biopsy. Re-HIFU based on the fused F-choline PET/MR images was performed, followed by a recurrence-free period of 11 months. Thus, F-choline PET/MR could improve guiding retreatment in patients with recurrence after HIFU.
\end{abstract}

DOI: https://doi.org/10.1097/RLU.0000000000001987

Posted at the Zurich Open Repository and Archive, University of Zurich ZORA URL: https://doi.org/10.5167/uzh-149939

Journal Article

Published Version

Originally published at:

Burger, Irene A; Gafita, Andrei; Müller, Julian; Kranzbühler, Benedikt; Donati, Olivio F; Eberli, Daniel (2018). 18F-Choline PET/MR Can Detect and Delineate Local Recurrence After High-Intensity Focused Ultrasound Therapy of Prostate Cancer. Clinical Nuclear Medicine, 43(4):e111-e112.

DOI: https://doi.org/10.1097/RLU.0000000000001987 


\title{
${ }^{18} \mathrm{~F}$-Choline PET/MR Can Detect and Delineate Local Recurrence After High-Intensity Focused Ultrasound Therapy of Prostate Cancer
}

\author{
Irene A. Burger, MD, * Andrei Gafita, MD, * Julian Müller, MD, * Benedikt Kranzbühler, MD, $\uparrow$ \\ Olivio F. Donati, MD, $\neq$ and Daniel Eberli, MD, PhD $†$
}

\begin{abstract}
Restaging local recurrence after high-intensity focused ultrasound (HIFU) is based on multiparametric MRI (mpMRI). However, postinterventional changes of the tissue, such as edema or hemorrhage, are limiting tumor detection on mpMRI. We present a case of a rising prostatespecific antigen values, negative mpMRI, and a Gleason score of $4+4$ on template biopsy after HIFU. On ${ }^{18} \mathrm{~F}$-choline PET/MR, high focal uptake was detected at the location of positive biopsy. Re-HIFU based on the fused ${ }^{18} \mathrm{~F}$-choline PET/MR images was performed, followed by a recurrence-free period of 11 months. Thus, ${ }^{18} \mathrm{~F}$-choline PET/MR could improve guiding retreatment in patients with recurrence after HIFU.
\end{abstract}

Key Words: HIFU, multiparametric MRI, prostate cancer, PSA, recurrence detection

(Clin Nucl Med 2018;43: e111-e112)

Received for publication November 6, 2017; revision accepted December 29, 2017.

From the Departments of *Nuclear Medicine and †Urology, and $\ddagger$ Institute of Diagnostic and Interventional of Radiology, University Hospital Zurich, University of Zurich, Zurich, Switzerland.

Conflicts of interest and sources of funding: none declared.

Correspondence to: Irene A. Burger, MD, Department of Nuclear Medicine, University Hospital Zurich, Rämistrasse 100, 8091 Zürich, Switzerland. E-mail: irene.burger@usz.ch.

Copyright (C) 2018 Wolters Kluwer Health, Inc. All rights reserved.

ISSN: 0363-9762/18/4304-e111

DOI: 10.1097/RLU.0000000000001987

\section{REFERENCES}

1. Blana A, Walter B, Rogenhofer S, et al. High-intensity focused ultrasound for the treatment of localized prostate cancer: 5-year experience. Urology. 2004; 63:297-300.

2. Poissonnier L, Chapelon JY, Rouviere O, et al. Control of prostate cancer by transrectal HIFU in 227 patients. Eur Urol. 2007;51:381-387.

3. Rouvière O, Girouin N, Glas L, et al. Prostate cancer transrectal HIFU ablation: detection of local recurrences using T2-weighted and dynamic contrast-enhanced MRI. Eur Radiol. 2010;20:48-55.

4. Muller BG, Fütterer JJ, Gupta RT, et al. The role of magnetic resonance imaging (MRI) in focal therapy for prostate cancer: recommendations from a consensus panel. BJU Int. 2014;113:218-227.

5. Schmid DT, John H, Zweifel R, et al. Fluorocholine PET/CT in patients with prostate cancer: initial experience. Radiology. 2005;235:623-628.

6. Giovacchini G, Picchio M, Coradeschi E, et al. [(11)C]choline uptake with PET/CT for the initial diagnosis of prostate cancer: relation to PSA levels, tumour stage and anti-androgenic therapy. Eur J Nucl Med Mol Imaging. 2008; 35:1065-1073.

7. de Perrot T, Rager O, Scheffler M, et al. Potential of hybrid ${ }^{18}$ F-fluorocholine PET/MRI for prostate cancer imaging. Eur J Nucl Med Mol Imaging. 2014; 41:1744-1755.

8. Piert M, Montgomery J, Kunju LP, et al. ${ }^{18} \mathrm{~F}$-choline PET/MRI: the additional value of PET for MRI-guided transrectal prostate biopsies. J Nucl Med. 2016; $57: 1065-1070$ 

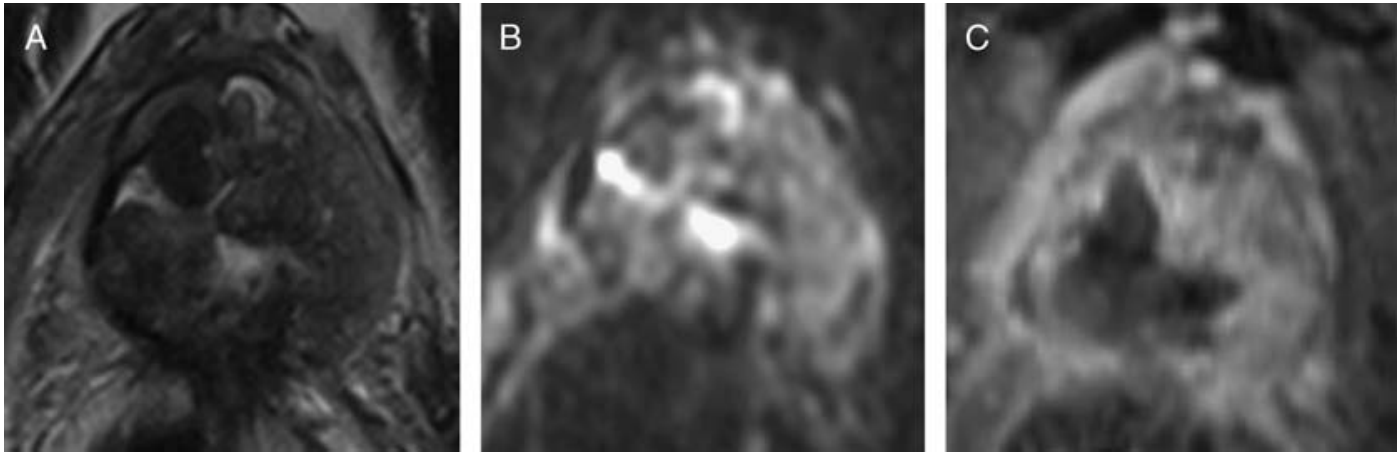

FIGURE 1. Multiparametric MRI (mpMRI) performed 6 months after high-intensity focused ultrasound (HIFU) due to a rising prostate-specific antigen (PSA) from nadir $1.6 \mathrm{ng} / \mathrm{mL}$ to $2.5 \mathrm{ng} / \mathrm{mL}$ and a positive template biopsy (Gleason score $4+4$ ). A, Axial T2-weighted image shows posttherapeutic changes in the right lobe of the prostate gland without any clear delineation of a focal lesion. B, Axial high $b$-value diffusion-weighted imaging (DWI) $(b=1400)$ showed no focal diffusion restriction, and $(C)$ the corresponding contrast enhanced scan showed no increased focal enhancement.
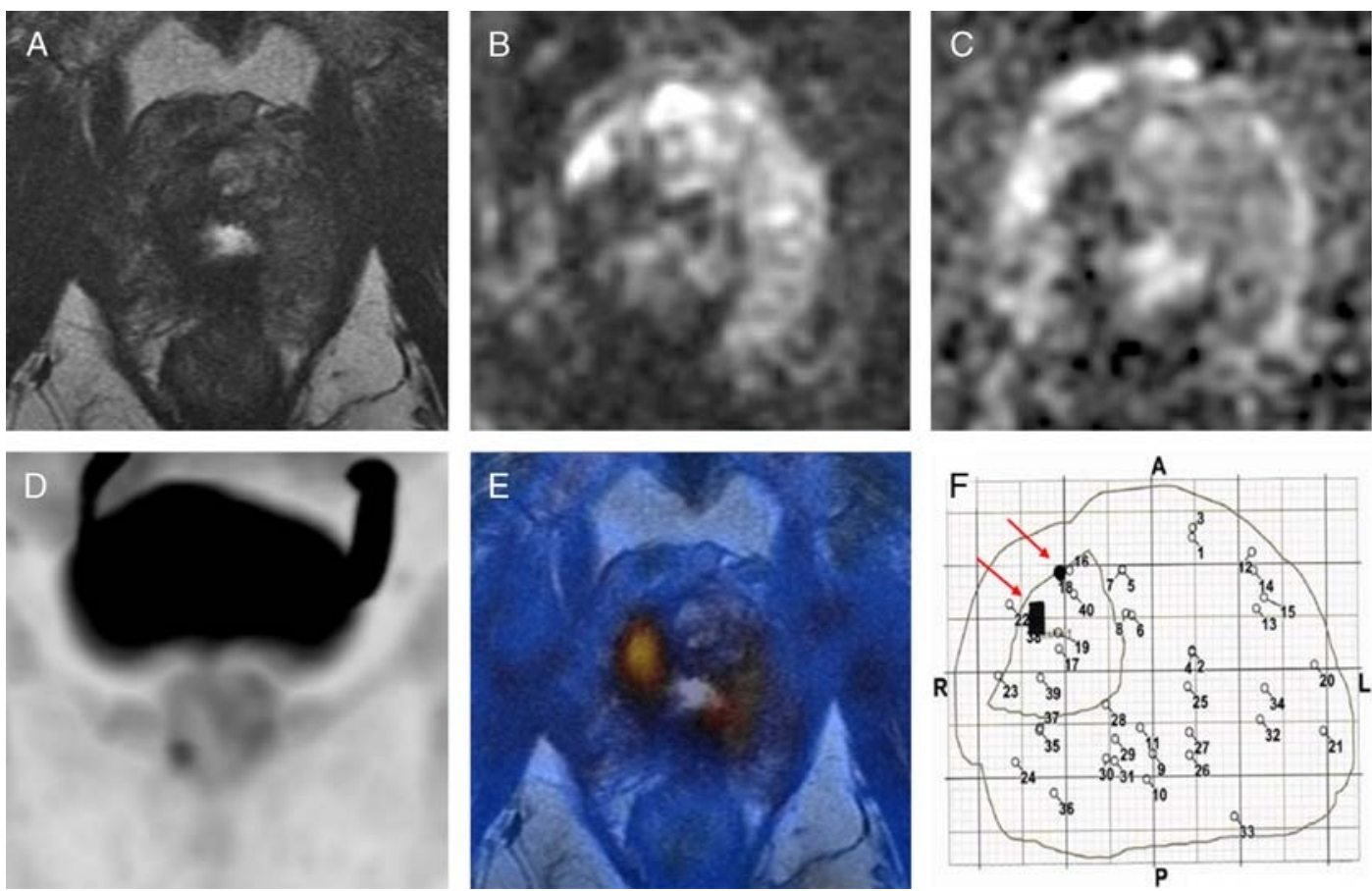

FIGURE 2. One month later, an ${ }^{18} \mathrm{~F}$-choline PET/MR was performed to reassess the prostate. Axial T2-weighted image (A) reveals a hypointense lesion within the right anterior lobe of the prostate, whereas diffusion images show a hypointense signal on both DWI image (B) and apparent diffusion coefficient map (C). Coronal ${ }^{18} \mathrm{~F}$-choline MIP image shows a clear focal uptake in the right peripheral lobe (D). Fused axial ${ }^{18} \mathrm{~F}$-choline PET with T2 MR confirms the focal choline-avid lesion within the right anterior prostate gland (SUVmax 4.5) (E), highly suggestive of malignancy, with good correlation to the cancer positive cores (red arrow) on the transperineal template biopsy that revealed a Gleason score $4+4(F)$. A re-HIFU was performed based on PET/MR findings, with falling PSA levels to $0.2 \mathrm{ng} / \mathrm{mL} 4$ months later. Five-year disease-free survival rates after HIFU ablation of clinically localized prostate cancer range between $66 \%$ and $78 \% .^{1,2}$ In patients with disease recurrence, tumor localization could facilitate further targeted treatment. To achieve this, mpMRI including perfusion images was suggested to increase the detection of significant tumor compared with unguided transrectal biopsy after HIFU. ${ }^{3}$ Some authors even suggest that template biopsy is not needed if a high-quality mpMRI is negative. ${ }^{4}$ In our experience, however, significant tumor found on template biopsy often lacks a clear correlate on mpMRI, including dynamic contrast-enhanced and DWI images. ${ }^{18} \mathrm{~F}$-choline PET is known to be positive in prostate cancer with a good sensitivity and impaired specificity due to intense uptake in benign prostate hyperplasia. ${ }^{5,6}$ However, it has been shown that the combination of ${ }^{18} \mathrm{~F}$-choline PET with MRI in hybrid systems can improve the specificity because benign prostate hyperplasia can be characterized with MRI. ${ }^{7,8}$ This is the first report showing that ${ }^{18}$ F-choline PET/MR could localize recurrence after HIFU with a good correlation to template biopsy. Furthermore, the tumor delineation allows planning a re-HIFU based on the PET uptake, with a very promising decrease in PSA of more than $90 \%$ after re-HIFU. 